\title{
Women's Reproductive Health in Sociocultural Context
}

\author{
Yael Benyamini $^{1} \cdot$ Irina Todorova $^{2}$ (D)
}

Published online: 17 November 2017

(C) International Society of Behavioral Medicine 2017

\begin{abstract}
Purpose The special issue on Women's Reproductive Health in Cultural Context covers multiple dimensions of women's reproductive health and how it might be shaped by cultural meanings, social and gender inequities, and power differentials, employing a variety of methodological approaches. On the one hand, it aims to bring to the forefront the conversation about how women's health is uniquely experienced and constructed in local settings, and on the other hand, it aims to draw broader conclusions from a perspective of interconnectedness of women and the shared issues that they face.

Methods and Results The special issue was initiated through a call for submissions and includes ten articles on the topic of women's reproductive health in cultural context.

Conclusions The articles provide many insights into how the context in which they live can disadvantage women and endanger their health, as well as offer perspectives on women's resistance to disempowering and stigmatizing discourses and practices. It aims to be of interest to scholars in behavioral medicine, psychology, and other social sciences
\end{abstract}

Irina Todorova

Irina_todorova@post.harvard.edu

Yael Benyamini

benyael@post.tau.ac.il

1 Bob Shapell School of Social Work, Tel Aviv University, 69978 Tel Aviv, Israel

2 Health Psychology Research Center, 1113 Sofia, Bulgaria

\section{Introduction}

The idea for this special issue emerged through our conversations and conference attendance during which we observed that the topic of women's health, which was more visibly present at our forums previously, has had a muted presence in health psychology and behavioral medicine in recent years. This observation is echoed also in other fields, which find a reduction of articles discussing women's health from a critical or social justice perspective [1]. While it certainly has not completely disappeared, it has been primarily interwoven into research on specific chronic diseases and reproductive health conditions and into studies of binary comparisons of men's and women's health outcomes. This has detracted from understanding the unique needs of women and girls in general and from the focus on the gendered, diverse, and contextual dimensions of women's health.

As it had observed 15 years ago, when it published a special issue on women's health [2], the International Journal of Behavioral Medicine is again highlighting the need for exploring unique questions relevant to women and their health in the contexts in which they live. Note that this special issue is focused on women's reproductive health in and of itself, not in comparison with men's health. Men are also embedded in and affected by socio-cultural norms and patriarchal structures in many, often negative ways. However, such an analysis is beyond the scope of this special issue.

The articles in this issue cover multiple dimensions of women's health and how these might be shaped by cultural meanings, social and gender inequities, and power differentials, employing a variety of methodological approaches. Thus, while some of the articles touch upon structural and financial barriers in access to health services, the emphasis is on how the specific contexts, cultural norms, and meanings of 
reproductive choices, constructions of femininity, motherhood, and womanhood are embodied and manifested in health consequences. This issue is based on respect for cultural traditions and uniqueness, while also encouraging an exploration of the potential ways in which existing norms can be problematic for health.

Some common themes can be identified in the articles encompassed by this issue. We see several examples of the medicalization of women's health and bodies, in which on the one hand the healthcare system constructs menstruation, pregnancy, and childbirth (or its absence) as medical conditions in need of treatment. At the same time, this reduces complex reproductive phenomena to biological processes, depriving women from the full range of choices potentially available to them and from the feelings of control over their bodies and their lives that comes with exercising choice. This can be accompanied with insensitivity to the experiential and emotional aspects of reproductive events [3] or with ignoring the multiple empowering or oppressive meanings surrounding them [4]. In other cases, communication is muted and women's concerns are silenced due to fear and concern for others [5], taboos about sexual topics and behaviors such as menstruation, sexuality [6], and condom use negotiation [7], with potential implications for well-being and safety.

Femininity and womanhood have specific cultural meanings and images, yet they are often conflated with motherhood, which is assumed to be the realization of the fundamental nature of women as nurturing. Motherhood is a respected, positive identity yet its dominant role in most cultures contributes to minimizing the diversity among women. Consequently, discourses regarding women continue to categorize them into binary groups of which one is "good" and the other is "evil" or "irresponsible" $[8,9]$, mother or childless $[10,11]$, maternal or sexual, and civilized or "primitive" [4]. One of the categories in these binaries is stigmatized - for example, the stigmatization of women who are not mothers either by choice or due to infertility is evident in many societies, since they do not fulfill the socially and personally expected role of a woman [12]. Such stigmatization and blame can have consequences for women's health through insensitivity to their distress, as well as through driving them to undergo endless cycles of fertility treatments $[10,11]$ which can ultimately endanger their health. Stigmatization of reproductive decisions or states is often coupled with paternalistic discourses regarding women as weak, uninformed, and needing protection or guidance - evident both among the public [4, 9], as well as among health providers [8].

Each of the articles underscores the importance of understanding women's health within the sociocultural context in which women live and which sets expectations and boundaries on their experience. Cultural expectations from women are not only externally imposed; they are often internalized by women, limiting their choice and control over their body and health. The range of topics in the current collection shows that this emphasis is relevant not only for studies of immigrant women or those from developing countries, or for examining controversial or stigmatized issues such as those related to abortion or HIV. Instead, the sociocultural context is indivisible from and fully permeates women's lives and experiences of health and illness. The articles in the issue provide multiple insights into how the above themes can disadvantage women and endanger their health, as well as offer perspectives on women's resistance to disempowering and stigmatizing discourses and practices.

Stankovic [3] interviewed Serbian women who gave birth for the first time in Serbia. She uncovered how this experience, usually constructed as "natural" and normative, is situated in local understanding and practices. Childbirth can be highly distressing within a public healthcare system that manages it without attending to women's needs and without involving them. Consequently, the experience of childbirth ranges from feelings of isolation and abandonment, lack of choice and control, to depriving women of their rights for information and consent to procedures. Childbirth is a pivotal experience in women's lives, with long-ranging consequences on maternal and child outcomes.

Taut [4] investigated views of public breastfeeding, as expressed in discussion forums in Romania. While breastfeeding is often medically recommended and presented as "natural," carrying it out in public drew ambivalent and opposing views. Her analysis reveals how different conflicts intersect around this issue: The idealization of breastfeeding and acknowledging women's right to choose (whether, when, and where to nurse); breastfeeding as a source of pride or shame and guilt; the breast as a natural way to feed a baby, who also has rights, or as a sexual organ that should be "disciplined" by society and hidden by mothers; the parallel between "natural" and "primitive" which leads to constructing women who breastfeed in public as being of lower-status.

Cultural differences play a prominent role in the studies that focused on immigrant women. Warmoth, Cheung, You, Yeung, and $\mathrm{Lu} \mathrm{[5]} \mathrm{used} \mathrm{expressive} \mathrm{writing} \mathrm{to} \mathrm{uncover} \mathrm{the}$ experiences of Chinese-American immigrant women who are breast cancer survivors. Even though all women had been living in the USA for years, negative cultural (mis)perceptions about the disease prevailed. Difficulties in disclosing their condition and expressing emotions led to barriers to seeking and receiving help, placing a heavy burden on the women. Their identities, deeply rooted in their home culture, clashed with the need to cope with the disease within a Western culture and healthcare system.

Drawing on interviews and focus groups with immigrant and refugee women living in Australia and Canada, Metusela et al. [6] investigated their experiences in relation to a sensitive topic, sexual and reproductive health. Their findings show 
how taboos about women's bodies and about sex permeate even close relationships, preventing open discussion that would allow women to obtain knowledge not only about safe sex, contraception, sexually transmitted diseases, and vaccinations, but even about processes such as menstruation and menopause. The end result was that many women had traumatic experiences of menarche, sex, pregnancies, and medical encounters.

Patrão and McIntyre [7] worked with women in Mozambique, also emphasizing how cultural norms could hamper women's attempts to practice safe sex for protection from HIV infection. A key issue is their ability to negotiate condom use with their partners. Those with greater confidence in doing so were younger, more educated, and independent, while many other women at risk for HIV infection had lower knowledge of HIV/AIDS and experienced many more barriers to safe sex in a context where women's lives are usually dominated by their partners.

While paternalizing and controlling social attitudes towards women are often equated with cultures in developing countries, similarly stronger forces can be found in pronatalist societies even in highly developed countries, pointing to the interconnectedness of women's health issues across contexts [13]. Two of the articles address women's difficulties in coping with infertility in Israel. While this would be considered "involuntary childlessness" in most countries, these articles emphasize how strong social pressure makes voluntary childlessness almost unthinkable in such a pronatalist country, leaving women undergoing infertility treatments in a very fragile situation. Neter and Goren [11] show how difficult it is to maintain well-being when the woman perceives the fertility problem as central to her identity and finds it difficult to disengage from related goals, or manages to disengage but not to reengage in other goals, leaving her with feelings of distress and emptiness.

Benyamini, Gozlan, and Weissman [10] provide further evidence of the quality of life costs of undergoing infertility treatments in a pronatalist culture. The women in their study struggled to maintain regular life routines and to feel "normal," as compared to their age peers. Standing up to these challenges more successfully enabled women to preserve well-being over time, but this was seen only among women who already had a child and thus were less exposed to social stigma.

Chiweshe and MacLeod [8] address an alternative reproductive choice for women which is often stigmatized - that of the decision to interrupt a pregnancy. They focus particularly on the way health care providers, who see women to provide post-abortion care, position these women as irresponsible, manipulative, ignorant, and immoral. In parallel, the authors identify the reflexive positions of the health providers of themselves, and illustrate how all of these are situated within cultural meanings and power relations. The implication of this negative positioning of women seeking care for the quality of the care they receive is discussed.

Discourses regarding abortion in Canada have shifted in the recent past as shown through the analysis conducted by Deurksen and Lawson [9]. Their contribution illustrates how the shift of these discourses from a focus on the fetus to that on the woman is accompanied by constructing women as misguided, unable to make decisions, and in need of protection. They extract the "benevolent sexism" and thus the continued stigmatization of women making such reproductive choices, hidden in this shift.

Granek and Nakash [12] provide an intriguing analysis of the ways in which social, historical, political, cultural, and geographic forces, including militarism and patriarchy, combine to create such heavy social pressure on Israeli (Arab and Jewish) women in all aspects of their reproductive health. Their analysis provides a reminder that social pressures, depriving women of full choice and control over their bodies can take place in any country and make use of technologically sophisticated medicalization to institutionalize social forces.

In summary, this special issue aims to bring to the forefront the conversation about how women's health is on the one hand uniquely experienced and constructed in local settings, and on the other to draw broader conclusions from a perspective of interconnectedness of women and the shared issues that they face [13], well as from the core understanding of health as a human right [14]. The issues raised here cannot be resolved solely by providing more information, to women or to health care providers; they require open communication with women, as subjects and not objects, and from an accepting and non-judgmental viewpoint. Moreover, the literature has also consistently illustrated the importance of women's health for sustaining the health of the whole population [15] as well as for the sustainable development of societies [16].

\section{References}

1. Bird M, Wright RL, Frost CJ. Enhancing women's health: a call for social work research. Soc Work Health Care. 2016;55(9):732-51. https://doi.org/10.1080/00981389.2016.1205706.

2. Powell LH, Matthews KA. New directions in understanding the link between stress and health in women. Int J Behav Med. 2002;9(3):173-5. https://doi.org/10.1207/S15327558IJBM0903 01.

3. Stankovic B. Women's experiences of childbirth in Serbian public healthcare institutions: a qualitative study. Int J Behav Med. 2017(This issue). https://doi.org/10.1007/s12529-017-9672-1.

4. Taut D. Breastfeeding (un) covered: narratives of public breastfeeding on Romanian discussion forums. Int J Behav Med. 2017(This issue). https://doi.org/10.1007/s12529-017-9687-7.

5. Warmoth K, Cheung B, You J, Yeung NCY, Lu Q. Exploring the social needs and challenges of Chinese American immigrant breast cancer survivors: a qualitative study using an expressive writing 
approach. Int J Behav Med. 2017(This issue). https://doi.org/10. 1007/s12529-017-9661-4.

6. Metusela C, Ussher J, Perz J, Hawkey A, Morrow M, Narchal R et al. "In my culture, we don't know anything about that": Sexual and reproductive health of migrant and refugee women. Int J Behav Med. 2017(This issue). https://doi.org/10.1007/s12529-017-96623.

7. Patrao AL, McIntyre TM. Socio-demographic, marital, and psychosocial factors associated with condom use negotiation self-efficacy among Mozambican women at risk for HIV infection. Int J Behav Med. 2017(This issue). https://doi.org/10.1007/s12529-017-96810 .

8. Chiweshe MT, Macleod C. "If you choose to abort, you have acted as an instrument of Satan": Zimbabwean health service providers negative constructions of women presenting for post abortion care. Int J Behav Med. 2017(This issue).

9. Duerksen KN, Lawson KL. "Not brain-washed, but heart-washed": a qualitative analysis of benevolent sexism in the anti-choice stance. Int J Behav Med. 2017(This issue). https://doi.org/10.1007/s12529017-9633-8.

10. Benyamini Y, Gozlan M, Weissman A. Normalization as a strategy for maintaining quality of life while coping with infertility in a pronatalist culture. Int J Behav Med. 2017(This issue). https://doi. org/10.1007/s12529-017-9656-1.

11. Neter E, Goren S. What happens while infertility treatments do not "deliver"? Association between the infertility centrality in the woman's identity, goal adjustment and psychological adjustment among women in ongoing fertility treatments. Int J Behav Med. 2017.

12. Granek L, Nakash O. The impact of militarism, patriarchy, and culture on Israeli women's reproductive health and well-being. Int J Behav Med. 2017(This issue). https://doi.org/10.1007/s12529017-9650-7.

13. Simon-Kumar R, MacBride-Stewart S, Baker S, Saxena LP. Towards north- south interconnectedness: a critique of gender dualities in sustainable development, the environment and women's health. Gend Work Organ. 2017; https://doi.org/10.1111/gwao. 12193.

14. Davidson PM, McGrath SJ, Meleis AI, Stern P, DiGiacomo M, Dharmendra T, et al. The health of women and girls determines the health and well-being of our modern world: a white paper from the international council on women's health issues. Health Care Women Int. 2011;32(10):870-86. https://doi.org/10.1080/ 07399332.2011 .603872 .

15. Davidson PM, Meleis AI. Sex and gender matters: the health of women and girls determines the health of our modern world. Health Care Women Int. 2016;37(8):817. https://doi.org/10.1080/ 07399332.2016.1218720.

16. Langer A, Meleis A, Knaul FM, Atun R, Aran M, Arreola-Ornelas $\mathrm{H}$, et al. Women and health: the key for sustainable development. Lancet. 2015;386(9999):1165-210. https://doi.org/10.1016/s01406736(15)60497-4. 